\title{
PERCEPÇÃO DA EQUIPE DE ENFERMAGEM QUANTO A DEAMBULAÇÃO PRECOCE NO PUERPÉRIO NA PREVENÇÃO DE TROMBOSE VENOSA PROFUNDA
}

\author{
PERCEPTION OF THE NURSING TEAM AS THE EARLY DEATHS IN THE PUERPÉRIO \\ IN THE PREVENTION OF DEEP VENOUS THROMBOSIS
}

\author{
BARBOSA, Debora Cibeli ${ }^{1}$; SILVA, Rosilaine Cristina da ${ }^{1}$, SOUZA, Silmara Alves de $^{2}$ \\ ${ }^{1}$ Graduando do Curso de Enfermagem da Universidade São Francisco - USF; ${ }^{2}$ Docente \\ Especialista do Curso de Enfermagem da Universidade São Francisco \\ silmaraas $a$ bol.com.br
}

RESUMO. A trombose venosa profunda (TVP) é o desenvolvimento de um coágulo dentro de um vaso sanguíneo, decorrente de uma inflamação ou trauma, determinando a obstrução total ou parcial do vaso. Diante de todas as ocorrências que pode haver no puerpério, a TVP é uma das grandes causas de complicações puerperais graves. A partir dessa realidade este trabalho teve como objetivo avaliar se a equipe de enfermagem da maternidade do Hospital Universitário do interior de São Paulo, tem como rotina a orientação com relação a deambulação precoce como prevenção da TVP. O trabalho tratou-se de um estudo descritivo, exploratório, de campo com abordagem qualitativa. Os resultados encontrados demonstraram que todos os profissionais da enfermagem sabem o que é a TVP e quais são os sinais e sintomas, porém não realizam as orientações quanto a deambulação voltada para a prevenção da TVP por desconhecimento do assunto. Portanto, faz-se necessária a capacitação da equipe de enfermagem quanto a orientação correta, para alcançar os objetivos da prevenção da TVP.

Palavras-chave: puerpério, trombose venosa profunda, deambulação, enfermagem.

ABSTRACT. Deep vein thrombosis (DVT) is the development of a clot within a blood vessel from inflammation or trauma, resulting in total or partial obstruction of the vessel. In view of all the occurrences that may occur in the puerperium, DVT is one of the major causes of severe puerperal complications. Based on this reality, this study had as objective to evaluate if the nursing team of the maternity hospital University Hospital, has as routine the orientation regarding early ambulation as prevention of DVT. The study was a descriptive, exploratory field study with a qualitative approach. The results showed that all nursing professionals know what DVT is and what the signs and symptoms are, but they do not carry out the guidelines regarding ambulation aimed at the prevention of DVT due to lack of knowledge of the subject. Therefore, it is necessary to train the nursing team on the correct orientation to achieve the objectives of DVT prevention.

Keywords: puerperium, deep vein thrombosis, ambulation. nursing.

\section{INTRODUÇÃO}

As gestantes podem apresentar no pós-parto sentimentos ambíguos como por exemplo euforia e angústia/ansiedade, confiança e medo, decepção e alegria que podem estar relacionados com o momento do parto, com a aparência e gênero do filho, com os medos e expectativas de ser uma boa mãe e necessidades do recém-nascido (MALDONADO, 2000). 
Apesar da preocupação com a humanização dos cuidados à saúde da mulher em todas as fases do ciclo vital sabe-se que o período puerperal tem pouca valorização, especialmente as relativas à subjetividade feminina em sua significação à maternidade. Os riscos de alterações fisiológicas e psicológicas na fase puerperal são considerados altos, portanto uma orientação qualificada da equipe de enfermagem é essencial para prevenir possíveis complicações físicas e emocionais. Essas orientações devem ser realizadas com empatia, acolhimento e tomando o cuidado em ouvir a puérpera com relação principalmente as expectativas sociais relativas à maternidade (STRAPASSON, NEDEL, 2010; ALMEIDA, SILVA, 2008; GONÇALVES, 2005).

Objetivos de Desenvolvimento do Milênio criado pela Organização Mundial da Saúde propõe melhora na saúde materna, porém ainda há pouco relato na literatura científica sobre os cuidados oferecidos à puérpera. A realidade presente nos serviços de saúde apresentados para a puérpera está muito distante do modelo assistencial preconizado pelas organizações de saúde, o que demonstra desinformação do profissionais da saúde sobre as reais necessidades das mulheres no período puerperal. A prestação de cuidado no puerpério é de vital importância para o bem-estar da mulher. A via de parto e a atenção dispensada ao puerpério imediato podem contribuir para o aparecimento de desconfortos que não estavam presentes no período gestacional ou mesmo agravar de queixas preexistentes (VANDERKUIK et al., 2013).

Diante de todas as ocorrências que pode haver no puerpério, a trombose venosa profunda (TVP) é uma das grandes causas de complicações puerperais graves (PEREIRA et al., 2011).

A TVP é uma obstrução total ou parcial do vaso em desenvolvimento de um coágulo dentro do mesmo, decorrente de uma reação inflamatória ou trauma, podendo levar ao tromboembolismo venoso, em que o coágulo se desloca pelo vaso. Se o quadro se agravar pode desenvolver uma embolia pulmonar, importante causa de morbidade e mortalidade (WHITE, 2003; ZANCHETTIN et al., 2011).

A incidência de TVP é de $0,36 \%$ a $0,6 \%$, durante a gravidez, se agravando de três a cinco vezes no período puerperal, estando entre $2,7 \%$ e $3 \%$. A embolia pulmonar é a primeira manifestação da TVP silenciosa dos membros inferiores, para a maioria dos casos, e ocorre em $0,27 \%$ e $1,2 \%$ dos eventos, uma vez não tratada pode levar a $15 \%$ de mortalidade (ANDRADE; VASCONCELOS, 2000).

A Sociedade brasileira de angiologia e cirurgia vascular adverte

\begin{abstract}
para o fato de que a trombose venosa precisa ser considerada uma complicação grave, porque sua instalação é silenciosa, o que retarda o diagnóstico e dificulta a intervenção por parte dos profissionais. Os sintomas da TVP se confundem com os desconfortos vasculares da gravidez, podendo ocorrer repercussões fetais quando se utiliza o tratamento com medicamentos anticoagulantes, além de sequelas vasculares que impedem a retomada das atividades diárias (SBACV, 2003).
\end{abstract}

A ausência da prática de métodos clínicos e complementares objetivados para o diagnóstico de TVP aumentam a incidência real de tais episódios trombóticos, porém se tais práticas fossem incorporadas ao cotidiano dos profissionais da saúde seriam de grande importância, pois, quando este tipo de trombo é corretamente diagnosticado e tratado, os riscos de embolia pulmonar e mortalidade materna diminuem consideravelmente (CLARK, 2001). 


\section{Schellong et al. (1999) refere que}

os efeitos potencialmente benéficos da mobilização precoce relacionam-se à teoria da bomba muscular da panturrilha e ao treino muscular. Durante a contração muscular, ocorre aumento na habilidade de ejeção, facilitando o retorno venoso, o que, por sua vez, reduz o gradiente de pressão hidrostática, responsável pela formação do edema, bem como melhora a perfusão muscular potencializando sua ação.

O estudo de Partsch et al. (2009) corroborou ao relatar que compressão externa da perna, aliada ao programa de caminhadas, promove redução na pressão sanguínea o que culmina na diminuição dos sinais e sintomas venosos.

A partir dessa realidade, elaboraram-se os questionamentos: a equipe de enfermagem está capacitada para detectar sinais, sintomas e/ou fatores de risco da TVP no atendimento puerperal? A capacitação exerce influência sobre essa prática? Que cuidados os enfermeiros identificam para os casos de TVP? Identificar os sinais e sintomas relacionados a TVP na percepção da equipe de enfermagem e apreciar os cuidados de enfermagem voltados à prevenção e controle da TVP sob o ponto de vista desses profissionais.

Portanto, o cuidado de enfermagem prestados no puerpério objetivam oferecer estratégias de enfrentamento e adaptação que facilitem a transição à maternidade, com atividades que irão diminuir possíveis sintomas puerperais (MOURA et al., 2007).

No Brasil e no mundo uma das causas de morbimortalidade entre as puérperas é a Trombose Venosa Profunda. Além dos problemas de comorbidades já instaladas nas pacientes como HAS, DM, problemas circulatórios e cardíacos, havendo um agravante devido a não orientação dada dos profissionais de saúde quanto ao estímulo de deambular precocemente no pós-parto. Recorrente da falta de conhecimento apresenta-se um despreparo no momento que ocorre o problema, com isso geram-se custos para as instituições com aumento de dias de internações, resultando disso alterações no emocional e no psicológico da puérpera que também entra em conflito. Gerando uma dificuldade na amamentação e nos cuidados básicos com recém-nascido.

Diante do exposto, este estudo teve como objetivo realizar a verificação do conhecimento da equipe de enfermagem, da maternidade de Hospital Universitário do interior de São Paulo, sobre a importância da deambulação precoce no puerpério como prevenção da trombose venosa profunda. Identificar se existe uma rotina para orientação quanto a deambulação no puerpério. Identificar as dificuldades para realizar as orientações para as puérperas.

\section{METODOLOGIA}

Tratou-se de um estudo descritivo, exploratório, de campo com abordagem qualitativa. A população de estudo foi composta por profissionais da equipe de enfermagem (enfermeiros, técnicos de enfermagem e auxiliares de enfermagem) que atuam diretamente com as puérperas na maternidade do Hospital Universitário no interior de São Paulo. Os critérios de exclusão para realização da coleta de dados foram os funcionários que se negaram a responder o questionário ou aqueles que estavam de licença médica, licença maternidade ou férias no período da coleta de dados.

Para a coleta de dados foi utilizado um questionário composto por perguntas abertas e fechadas referentes ao conhecimento da equipe em relação as orientações para deambulação 
precoce no puerpério elaborado e aplicado pelas pesquisadoras. Este questionário foi elaborado utilizando informações contidas em bases de dados como Literatura LatinoAmericana e do Caribe em Ciências da Saúde (LILACS), a biblioteca eletrônica Scientific Electronic Library Online (SCIELO), livros e revistas através dos seguintes descritores: trombose venosa profunda, deambulação, puerpério, enfermagem.

Os dados foram analisados estatisticamente e apresentados de forma gráfica, com números percentuais e absolutos. Os mesmos foram agrupados para discussão, comparandoos com a literatura. Este trabalho teve a aprovação do Comitê de Ética CAEE $\mathrm{n}^{\circ} 89382118.9 .000 .5514$.

\section{RESULTADOS E DISCUSSÃO}

Neste trabalho foram entrevistados 16 profissionais da enfermagem, entre auxiliares de enfermagem, técnico de enfermagem e enfermeiros. De acordo com os dados da tabela 1 dos entrevistados $25 \%$ eram enfermeiros, $62,5 \%$ eram técnicos de enfermagem e $12.5 \%$ auxiliares de enfermagem. Os trabalhadores entrevistados atuam no campo da saúde onde estão inseridos expressivamente em um hospital fortemente atuante no SUS, na educação e no ensino.

A enfermagem hoje no país é composta por um quadro de $80 \%$ de técnicos e auxiliares e $20 \%$ de enfermeiros, sendo que a área de saúde se compõe de um contingente de 3,5 milhões de trabalhadores, dos quais cerca 50\% atuam na enfermagem. O perfil da enfermagem, estimada em 50\% dos municípios brasileiros e em todos os 27 estados da Federação, inclui desde profissionais no começo da carreira (auxiliares e técnicos, que iniciam com 18 anos de idade; e enfermeiros, com 22 anos) até os aposentados (pessoas de até 80 anos). A equipe de enfermagem é predominantemente feminina, sendo composta por $84,6 \%$ de mulheres. É importante ressaltar, no entanto, que mesmo tratando-se de uma categoria feminina, registra-se a presença de $15 \%$ dos homens. A masculinização da enfermagem está crescendo juntamente com a demanda de trabalhos ofertadas pela categoria. No quesito mercado de trabalho, 59,3\% das equipes de enfermagem encontram-se no setor público; $31,8 \%$ no privado; $14,6 \%$ no filantrópico e $8,2 \%$ nas atividades de ensino (COFEN, 2015).

Segundo Brasil (2015) mais da metade dos enfermeiros (53,9\%), técnicos e auxiliares de enfermagem $(56,1 \%)$ se concentram na Região Sudeste. Proporcionalmente à população, que representa $28,4 \%$ dos brasileiros, a Região Nordeste apresenta a menor concentração de profissionais, com $17,2 \%$ das equipes de enfermagem. O Enfermeiro ainda possui uma contingência baixa em todo o país, e os Técnicos de Enfermagem continua sendo um número expressivo no território nacional (COFEN, 2015).

Quanto ao tempo de trabalho na instituição onde a pesquisa foi desenvolvida dentre os entrevistados $6,25 \%$ dos profissionais possuía 5 anos dentro da instituição, $50 \%$ possuía 10 anos de instituição e $43,75 \%$ possuíam até 25 anos de instituição. E o tempo de trabalho no setor da maternidade $37,5 \%$ dos profissionais possuíam 2 anos de colocação e $62,50 \%$ possuíam 10 anos de colocação no setor (Tabela 1).

Até meados da década de 1850, a enfermagem era uma profissão voltada à modéstia, altruísmo, humildade e disciplina. Contudo apesar de na sociedade atual a enfermagem ser reconhecida como ciência, muitas pessoas creem que para exercê-la precisa-se de um dom natural e não de estudo, conhecimento científico e aprofundado, tanto que esta crença popular interfere no reconhecimento profissional e salarial desta classe tão importante dentre os profissionais da saúde (WISNIEWSKI et al., 2015; AVILA et al., 2013). 
Tabela 1 - Caracterização da amostra estudada segundo categoria profissional, tempo de trabalho na instituição de estudo e tempo de trabalho no setor de atuação $(\mathrm{N}=16)$.

\begin{tabular}{lll}
\hline Categoria profissional & $\mathrm{N}$ & $\%$ \\
\hline Enfermeiro & 04 & 25,00 \\
Técnico de Enfermagem & 10 & 62,50 \\
Auxiliar de enfermagem & 02 & 12,50 \\
\hline Tempo de trabalho na instituição & $\mathrm{N}$ & $\%$ \\
\hline Menor que 5 anos & 01 & 6,25 \\
Entre 6 à 10 anos & 08 & 50 \\
Entre 11 à 25 anos & 07 & 43,75 \\
\hline Tempo de trabalho no setor & $\mathrm{N}$ & $\%$ \\
\hline Menor que 2 anos & 06 & 37,5 \\
Maior que 3 anos & 10 & 62,5
\end{tabular}

Fonte: Próprio autor

Na enfermagem, devido a subdivisão em diferentes níveis de conhecimento, ocorre na maioria das vezes uma distribuição hierárquica das tarefas, sendo que o enfermeiro fica responsável por responder pela equipe, supervisionar e controlar o trabalho executado pela equipe, sendo o responsável pela parte intelectual da equipe, contudo ele também exerce funções que envolvem esforço físico. Já os técnicos e auxiliares de enfermagem são os executores de tarefas mais técnicas que envolvem esforço físico (MAGNAGO et al., 2010).

Através dessas informações percebesse que por mais que a enfermagem tenha ganhado espaço na área da saúde e um processo de hierarquização continua sendo considerada por muitos uma profissão de benevolência e caridade. Porém, não se deve esquecer que sem este processo de assistência prestado ao paciente pela equipe de enfermagem muitas enfermidades não seriam sanadas pura e simplesmente por todo o aparato tecnológico disponível nos dia de hoje (WISNIEWSKI et al., 2015; AVILA et al., 2013; MAGNAGO et al., 2010).

$\mathrm{Na}$ tabela 2 é possível observar os dados coletados sobre o conhecimento dos profissionais entrevistados com relação a TVP e 100\% deles relatam conhecer sim a patologia, e a identificação dos sinais e sintomas que são: dor, calor rubor, edema, inchaço, câimbras e vermelhidão.

A TVP normalmente ocorre em pessoas sem nenhum histórico ou predisposição genética, porém algumas situações aumentam a chance de seu desenvolvimento, como por exemplo problemas fisiológicos - diminuição da atividade fibrinolítica e hipercoagulabilidade -, problemas de imobilidade - devido operações e traumas, viagens longas-, além de fatores pré-existentes - doenças malignas, idade avançada, falência cardíaca, obesidade, puerpério, uso de hormônios femininos, dentre outros (SILVEIRA, 2009). 
Tabela 2- Conhecimento da amostra estudada em relação a Trombose Venosa Profunda (TVP) e quanto a identificação de seus sinais e sintomas. Bragança Paulista, 2018 (N = 16).

\begin{tabular}{lll}
\hline Conhecimento sobre TVP & N & $\%$ \\
\hline Sim & 16 & 100 \\
Não & 00 & 00 \\
\hline Identificação de sinais e sintomas & N & $\%$ \\
\hline Sim & 16 & 100 \\
Não & 00 & 00 \\
\hline
\end{tabular}

Fonte: Próprio autor

Sendo assim, uma avaliação criteriosa realizada pela equipe de enfermagem é importante para detectar precocemente os sinais iniciais de distúrbios venosos das extremidades inferiores. A TVP, se encaixando dentro desses distúrbios, é diagnóstica se a equipe de enfermagem realizar com atenção os sinais e sintomas como anamnese e exame físico das puérperas, uma vez que o puerpério já é uma condição predisponente para essa patologia (SILVEIRA, 2009; SMELTZER, BARE, 1993).

Junqueira et al. (2006) e Cavalcante et al. (2003) revelam que gravidez aumenta o risco pela elevação dos fatores de coagulação e da tríade de Virchow - que é a estase venosa, lesão vascular e hipercoagulabilidade -, sendo agravada pela não-deambulação no pós-parto, o que causa estase sanguínea.

Sinais que aparecem devido ao movimento e que indicam possível TVP. Portando, a execução de teste/exame pela equipe de enfermagem é importante, tais como sinal de Homans (Homan/Holmans) que é caracterizado com a dor na panturrilha durante a dorsiflexão do pé sobre a perna, sinal de Bandeira que é quando se mobiliza as duas panturrilhas, a acometida apresenta menor mobilidade, sinal de Bancroft que é dor na musculatura da panturrilha à palpação (SBACV, 2003).

Normalmente a dor decorrente da trombose venosas de membro inferior é limitada a panturrilha, mas quando ocorre o acometimento em veias mais profundas a dor é mais branda e difusa, apresentando na forma de queimação, cãibra ou sensação de peso nas pernas, sendo amenizada quando se encontra no estado de repouso. Portanto, as execuções dos testes/exames citados acima auxiliam na detecção precoce da TVP. Além disto, a presença de edema unilateral ou assimétrico é o sinal/sintoma mais característico da TVP (ANDRADE et al., 2009, CAVALCANTE et al., 2003).

Além do sinal do edema de membro, outro sinal característico é o sinal da bandeira que ocorre devido a uma rigidez na musculatura da panturrilha que é percebida principalmente com o joelho fletido. Em casos mais graves onde há trombose venosa extensa, atingindo inclusive as vênulas e capilares venosos, aparece o quadro de phlegmasia cerulea dolens, que se caracteriza por cianose, petéquias, diminuição de pulsos e síndrome compartimental. Do ponto de vista geral, pode ser observado aumento da temperatura do membro afetado, ocorrer febre baixa e mal-estar inespecífico. Em casos mais graves pode ocorrer choque, gangrena venosa e até mesmo amputações (MAFFEI et al., 2001).

Quando os profissionais foram questionados com relação ao conhecimento que a deambulação tardia pode causar a TVP no puerpério 93,75\% responderam não conhecer e $6,25 \%$ responderam conhecer. Sobre a existência de rotina para a deambulação $93,75 \%$ profissionais responderam que realizam e $6,25 \%$ responderam não realizar. Em sua totalidade 
os profissionais relataram que a rotina realizada mantém-se para todos os partos realizados normal, fórceps, cesárea no tempo mínimo de $2 \mathrm{~h}$ a $8 \mathrm{hs}$ para deambulação (Tabela 3 ).

Tabela 3- Conhecimento da amostra estudada quanto ao fato da deambulação tardia ser um fator importante para causar Trombose Venosa Profunda (TVP) e a existência de rotina para a orientação das puérperas quanto a deambulação precoce. Bragança Paulista, $2018(\mathrm{~N}=16)$.

\begin{tabular}{lcc}
\hline $\begin{array}{l}\text { Conhecimento que deambulação tardia pode } \\
\text { causar a TVP? }\end{array}$ & $\mathrm{N}$ & $\%$ \\
\hline Sim & 01 & 6,25 \\
Não & 15 & 93,75 \\
\hline Existência de rotina para deambulação precoce & $\mathrm{N}$ & $\%$ \\
\hline Sim & 15 & 93,75 \\
Não & 1 & 6,25 \\
\hline Rotina relatada & $\mathrm{N}$ & $\%$ \\
\hline Parto normal: 2 horas pós-parto & 16 & 100 \\
Parto fórceps: 2 horas pós-parto & 16 & 100 \\
Parto cesárea: 8 horas pós-parto & 16 & 100
\end{tabular}

Fonte: Próprio autor

A equipe de enfermagem pode atuar na prevenção e tratamento da TVP através de métodos não invasivos, principalmente, e complementada pela organização de propostas educacionais, contribuindo assim para que a utilização dos métodos invasivos se restrinja às indicações (ZAMPIERI, 2002).

Bates et al. (2008) refere que nas puérperas sem história anterior de evento tromboembólico e submetidas à cesariana, o risco de tromboembolismo é maior do que naquelas submetidas ao parto normal.

Além disto, autores relatam que a equipe de enfermagem necessita obter mais conhecimentos através de educação e saúde, para uma melhor avaliação dos riscos inerentes do pós-parto (BATES et al., 2008; ZAMPIERI et al., 2002). Portanto, é de suma importância que a equipe de enfermagem tenha sempre a possibilidade de participar de eventos educacionais da área de atuação, para que o conhecimento chegue até todos os profissionais, para que os sinais e sintomas não passem despercebidos e/ou que a técnica seja desenvolvida sem o conhecimento do porquê está sendo executada. Estes fatores são importantes, uma vez que um bom exame possibilita o diagnóstico precoce e o não agravamento do quadro do paciente, diminuindo assim o custo para o sistema de saúde e o risco de infecções.

A última informação analisada juntos aos profissionais foi se havia dificuldade da equipe quanto a orientação da deambulação precoce e $87,5 \%$ dos profissionais disseram orientar as pacientes quanto a deambulação precoce, mas não é direcionada para a prevenção de TVP. E um profissional relatou não existir rotina para orientação para a deambulação. Outro profissional relatou que existe a rotina, mas não orienta. E ninguém alegou falta de tempo para orientar e auxiliar na deambulação. 


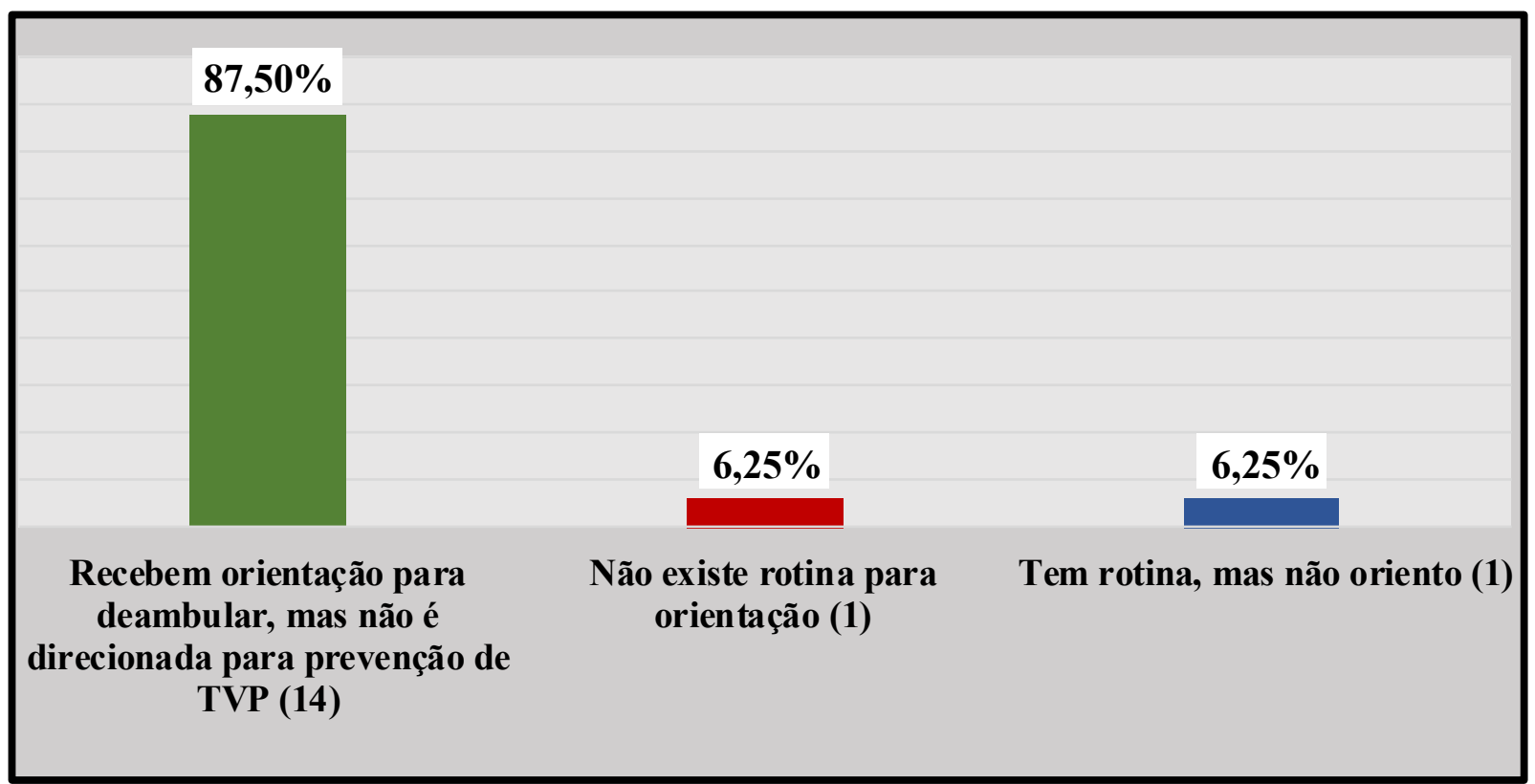

Figura 1- Gráfico da distribuição das dificuldades da equipe de enfermagem para realizar as orientações referentes à deambulação para as puérperas. Bragança Paulista, $2018(\mathrm{~N}=16)$ (Fonte: ASCHWANDEN et al. 2001).

durante muito tempo, preconizou-se repouso absoluto no leito como tratamento da TVP aguda. O protocolo comumente utilizado na prática hospitalar consiste em repouso, elevação de membros inferiores e anticoagulantes até que se obtenha a estabilidade do trombo. Entretanto, muitos estudos sugerem ser a deambulação precoce recomendada para a maioria dos pacientes com TVP, devendo haver maior precaução com indivíduos com histórico de embolia pulmonar prévia.

O exame físico e anamnese realizados pela equipe de enfermagem são importantes para a identificação precoce de agravante que culminem no agravamento do quadro clínico do paciente (ROMERO, 2010). Ao detectar os problemas, a prescrição de enfermagem torna-se fator determinante para o bom desenvolvimento dos cuidados e prognóstico pertinentes à saúde do indivíduo (BASTO et al., 2011).

Os autores como Basto et al. (2011) e Romero (2010), são categóricos em afirmar que a equipe de enfermagem necessita receber mais orientações quanto a deambulação precoce da TVP no que diz respeito ao puerpério.

\section{CONCLUSÃO}

Os resultados encontrados na pesquisa demonstraram que todos os funcionários da enfermagem entrevistados detêm o conhecimento com relação a TVP e aos seus sinais e sintomas. Contudo, a mesma pesquisa demonstrou que quase $94 \%$ dos profissionais entrevistados não sabem que procedimento de deambulação precoce é uma forma de prevenir o desenvolvimento da TVP. E que 93,75\% dos entrevistados relataram fazer a orientação com relação a deambulação, mas não é direcionada à prevenção da TVP, e 6,25\% não fazem a orientação. 
Conclui-se que a equipe de enfermagem da maternidade do Hospital Universitário estudado não obtém conhecimento suficiente a respeito da prevenção da TVP a partir da deambulação precoce. Por outro lado, espera-se que este estudo possa contribuir para uma maior atenção e cuidados básicos, e de orientações as puérperas. Faz-se necessária a capacitação da equipe de enfermagem quanto a orientação correta, para alcançar os objetivos da prevenção da TVP no puerpério, diminuindo assim a mortalidade materno-puerperal e o tempo de internação.

\section{REFERÊNCIAS}

ALMEIDA, M. S; SILVIA, I. A. Necessidades de mulheres no puerpério imediato em uma maternidade pública de Salvador. Rev. Esc. Enferm. USP. 42(2): 347-354, 2008.

ANDRADE, B. A. M.; GAGLIARDO, G. I.; PÉRET, F. J. A. Tromboembolismo venoso no ciclo gravídico puerperal. FEMINA. 37(11):611-618, 2009.

ANDRADE, J. A.; VASCONCELOS, L. A. O. Patologia cardíaca da gestação: abordagem e conduta na trombose venosa profunda (TVP) durante à gravidez e no pós-parto.

Patologias cardíacas da gestação. São Paulo: Edusp, p.135-148, 2000.

ASCHWANDEN, M.; LABS, K. H.; ENGEL, H.; SCHWOB, A.; JEANNERET, C.; MUELLER-BRAND, J.; JAEGER, K. A. Acute deep vein thrombosis: early mobilization does not increase the frequency of pulmonary embolism. Thromb Haemost. 85:42-46, 2001.

AVILA, L. I.; SILVEIRA, R. S.; LUNARDI, V. L.; FERNANDES, G. F. M.; MANCIA, J. R.; SILVEIRA, J. T. Implicações da visibilidade da enfermagem no exercício profissional. Rev Gaúcha Enferm. 34(3):102-109, 2013.

BASTOS, M.; BARRETO, S. M.; CAIAFA, J. S.; REZENDE, S. M. Tromboprofilaxia: recomendações médias e programas hospitalares. Revista da Associação Médica Brasileira. 57(1):88-99. 2011.

BATES, S. M.; GREER, I. A.; PABINGER, I.; SOFAER, S.; HIRSH, J. Venous thromboembolism, thrombophilia, antithrombotic therapy, and pregnancy: American College of Chest Physicians evidence-based clinical practice guidelines (8th edition). Chest. 33:6 Suppl:844S-886S, 2008.

BRASIL. IBGE - INSTITUTO BRASILEIRO DE GEOGRAFIA E ESTATÍSTICA. Estimativas populacionais para os municípios e para as Unidades da Federação brasileiros. IBGE 2015.

CAVALCANTE, M. B.; ALENCAR JÚNIOR, C. A.; ALMEIDA, F. M. Fatores de risco para tromboembolismo na gestação. Rev. Femina. (31): 819-21, 2003.

COFEN - CONSELHO FEDERAL DE ENFERMAGEM. Pesquisa inédita traça perfil da enfermagem. Cofen 2015. 
CLARK, S. L. Tratamento intensivo em Obstetrícia. $3^{a}$ ed., São Paulo: Livraria Santos Editora, 2001.

GONÇALVES, A. C. A puérpera e o recém-nascido em alojamento conjunto. In: Oliveira DL organizadora. Enfermagem na gravidez, parto e puerpério: notas de aula. Porto Alegre: Editora da UFRGS; p. 367-386, 2005.

JUNQUEIRA, M. S. R.; TONANI, L. L.; RIBEIRO, F. S.; LEITE, J. M.; SOARES, P. C.; SOUZA, J. H. K. Doenças tromboembólica na gestação. Rev. Med. Minas Gerais. 16(3): 170-173, 2006.

MAFFEI, F. H. A.; CAIAFA, J. S.; RAMACCIOTTI, E.; CASTRO, A A. Para grupo de elaboração de normas de orientação clínica para prevenção, diagnóstico e tratamento da trombose venosa profunda. Belo Horizonte: SBACV; 2001.

MAGNAGO, T. S. B. S.; LISBOA, M. T. L.; GRIEP, R. H.; ZEITOUNE, R. C. G.; TAVARES, J. P. Condição de trabalho de profissionais da enfermagem: avaliação baseada no modelo demanda-controle. Acta paul. Enferm. 23(6): 811-817, 2010.

MALDONADO, M. T. Psicologia da gravidez: parto e puerpério. $16^{\text {a }}$ ed. São Paulo: Saraiva; 2000

MOURA, F. M. J. S. P.; CRIZOSTOMO, C. D.; NERY, I. S.; MENDONÇA, R. C. M.; ARAÚJO, O. D.; ROCHA, S. S. A humanização e a assistência de enfermagem ao parto normal. Rev Bras Enferm. 60(4):452-455, 2007.

PARTSCH, H. Ambulation and compression after deep vein thrombosis: dispelling myths. Semin Vasc Surg.18:148-52, 2005.

PEREIRA, M. A. M.; GOMES, P. G. M.; MADUREIRA, B. A. N.; BERSAN, N. P.; VILELA, V. R.; SILVA, R. S.; MARTINS, A. S. T.; KRETTLI, C. S. W. Trombose venosa profunda proximal no puerpério. Rev. Med. Minas Gerais. 21(4 SUPL6)S1-S143, 2011.

ROMERO, M. N. Prevenção da Trombose Venosa Profunda em Pacientes Críticos. SETHGF. Capitulo 118; 129-140, 2010.

SILVEIRA, P. R. Trombose venosa profunda e gestação: aspectos etiopatogênicos e terapêuticos. J Vasc Bras. 1(1): 65-70, 2002.

SBACV - SOCIEDADE BRASILEIRA DE ANGIOLOGIA E CIRURGIA VASCULAR (BR). Protocolo de prevenção da trombose venosa profunda: recomendações para a prática clínica. Normas de apresentação tabular. Rio de Janeiro (RJ); 2003.

SCHELLONG, S. M.; SCHWARZ, T.; KROPP, J.; PRESCHER, Y.; BEUTHIENBAUMANN, B.; DANIEL, W. G. Bed rest in deep venous thrombosis and the incidence of scintigraphic pulmonary embolismo. Thromb Haemost. 82(suppl 1):127-129, 1999. 
SMELTZER, S. C.; BARE, B. G. Tratado de enfermagem médico cirúrgico de Brunner e Suddart.7 ed., Rio de Janeiro: Guanabara Koogan;1993.

STRAPASSOM, M. R.; NEDEL, M. N. B. Puerpério imediato: desvendando o significado da maternidade. Rev. Gaúcha. Enferm. 31(3):521-528, 2010.

VANDERKUIK, R. C.; TUNÇALP, O; CHOU, D. SAY, L. Framing maternal morbidity: WHO scoping exercise. BMC Pregnancy Childbirth. 13:213, 2013.

WHITE. R. T. The epidemiology of venous thromboembolism. Circulation. 107(23 Suppl 1):14-18, 2003.

WISNIEWSKI, D.; SILVA, E. S.; ÉVORA, Y. D. M.; MATSUDA, L. M. Satisfação profissional da equipe de enfermagem $x$ condições e relações de trabalho: estudo relacional. Texto contexto - enferm. 24(3):850-858, 2015.

ZAMPIERI, M. M. F. Manejo na Assistência à gestante de alto risco. Rev. Nursing. (48):22, 2002.

ZANCHETTIN, S. D,; GARLET, M. S.; OLIVEIRA, V. A.; HERMEL, P. P.; AMESTOY, S. C. Trombose venosa profunda e a importância da sistematização da assistência de enfermagem. In: XX Congresso de Iniciação Científica UFPEL; 2011.

Publicado em 29/10/2020 